\title{
Explore the Blended Teaching Model from the Perspective of Cognitive Load
}

\author{
Yingying Wei \\ Department of Management, East University of Heilongjiang, Harbin 150066, China \\ College of Mechanical and Electrical Engineering, Harbin Engineering University, 150001 Harbin, \\ Heilongjiang, China \\ nefuwyy@126.com
}

Keywords: Cognitive load; Blended teaching model; Interaction process; Knowledge presentation

\begin{abstract}
In order to solve the problem of cognitive overload, this paper analyzes the effects of blended teaching model on the learners' cognitive load, which based on cognitive load theory and information theory. The purpose of this study is to establish the "Knowledge-Cognition" interaction process and to explore the control strategy of cognitive load in the implementation of the blended teaching model. The results show that the discrete knowledge can be transmitted to the learners through the structured knowledge presentation system after careful editing by the instructional designer, which affects the learning experience and the knowledge acquisition effect together with the learning environment and the learning task. On the another hand, the problem of high or too low cognitive load in blended teaching model can be balanced through the construction of graphics information space, information prominence and filtering of secondary information.
\end{abstract}

\section{Introduction}

Blended teaching model is an in-depth "online” and "offline” teaching method through combining flipped classroom model (FCM), massive open online courses (MOOCs), micro-learning and small-scale private online courses (SPOCs) with traditional face-to-face educational methods. It greatly enhances the way learners can access high-quality teaching resources and meets the needs of diverse and individualized talent development.

With the increasing maturity of multiple teaching modes, in the classroom teaching process, some problems of courses are gradually exposed, such as excessive information, complex knowledge structure, boring teaching methods, and inability to interpret complex knowledge points in time [1]. The frustration of learners has become increasingly prominent.

According to educational psychologists, the difficulty in acquiring learners' knowledge during the teaching process is mainly reflected in the cognitive overload from the information processing process. Therefore, how to construct a blended teaching model in colleges from the theoretical perspective of cognitive load, while helping learners to learn effectively, pay attention to their cognitive tolerance and learning interest. It will become the mainstream direction of applied education reform and innovation in colleges and universities in the future.

\section{Cognitive Load Theory}

Cognitive Load Theory (CLT) refers to the effort being used in the working memory. It was first proposed by Australian cognitive psychologist John Sweller in 1988 [2]. He argued that instructional design can be used to reduce cognitive load in learners. Cognitive load differentiates into three types: intrinsic, extraneous, and germane [3].

Intrinsic cognitive load depends mainly on the difficulty of learning materials and learners' prior knowledge. The number, types, and complexity of the learning materials are fixed and cannot be changed by external factors. But learners can use the brain to establish schema associations with learning materials to reduce the internal cognitive load. The extraneous cognitive load is a 
psychological activity that does not contribute to learning. It is mainly manifested in the fact that the teaching designer uses inappropriate information presentation methods or arranges inappropriate teaching activities during the learning process. The learner does not directly produce the working memory effect when constructing the knowledge information schema or generates the interference effect when establishing the associated information schema, which will lead to additional cognitive load. Germane cognitive load refers to the load associated with facilitating schema construction and schema automation processes. It promotes learners to better allocate cognitive resources to effective activities by comparing, reasoning, packaging, and reorganizing scattered information resources, while an external cognitive load is a hindrance to learners' learning.

The boring or irritating behavior of most learners in acquiring knowledge is mainly caused by the cognitive load of the brain when processing information is too low or too high. The problem faced by the hybrid teaching model is, in the final analysis, the problem of cognitive load imbalance in the process of learner knowledge acquisition. From the perspective of balanced information presentation and learner cognitive load, we should study the influencing factors of cognitive load and adjust the teaching curriculum design mode in time to truly improve the online teaching effect and promote learners to actively and effectively acquire online teaching resources.

\section{Knowledge-Cognition interaction process}

The process of knowledge transfer is the process of information communication from the original discrete knowledge to the learners' cognitive memory. Based on Claude E. Shannon's information theory [4], this process can be divided into four aspects: discrete knowledge to knowledge collation source coding, knowledge presentation channel coding, perceptual channel decoding, brain knowledge construction or reconstructed source decoding. Among them, the interaction process of "Knowledge-Cognition" is influenced by the characteristics of learning materials, the characteristics of learning environment, the characteristics of learning tasks, and the characteristics of learners. As shown in Fig.1, the Knowledge-Cognition interaction process of blended teaching model is established.

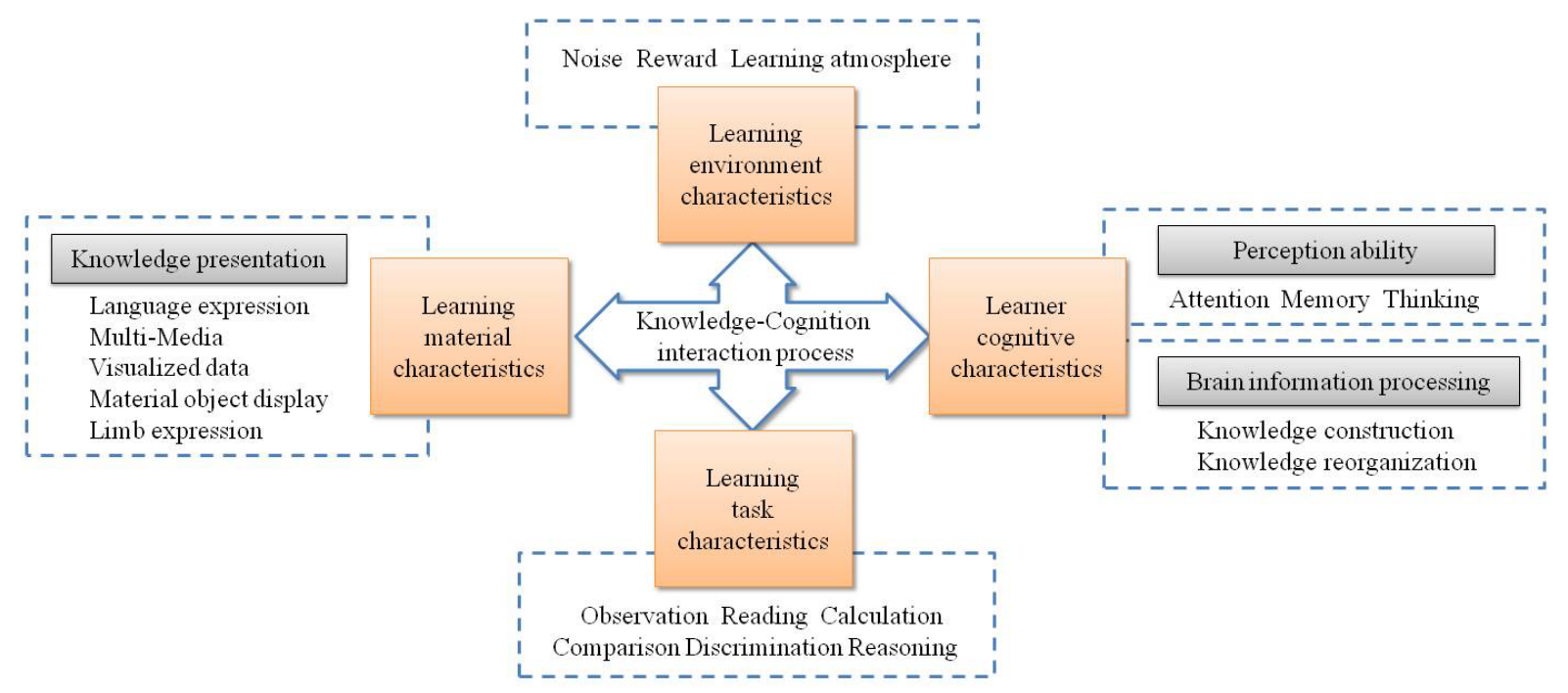

Fig.1 Knowledge-Cognition interaction process of blended teaching model

\section{Effects of blended teaching model on learners' cognitive load}

The micro-courses in animated form accord with the learner's internal cognitive load. The micro-class mainly presents the "fragmented" learning process, usually delivering specific knowledge content to specific target groups. The micro-class designers use the split explanation method to split the large-capacity information into a small series of classroom teaching videos, and the video duration is generally 5-8 minutes. Learners can select learning resources that meet the 
individual's internal cognitive load at any time through the mobile phone mobile terminal and can quickly expand the knowledge and cognitive field. For example, NetEase OpenCourses are mostly based on micro-courses, which are often used in the form of animation to teach professional knowledge, which stimulates interest in learning. The extra-curricular video "blockchain 100 questions" is used as the survey object, and 96 students spend an average of 2.47 hours. The 79 classes of micro-classes are all read and can tell the relevant content of the blockchain, which fully verifies that the micro-courses in the form of animation meet the learner's internal cognitive load.

Blended use of multiple teaching platforms produces high external cognitive load. With the rise of multimedia information teaching methods, the online and offline mobile network teaching mode has become the teaching method for the normalization of many applied universities. Each teacher will find a teaching platform that meets the characteristics of their respective courses, such as Blue ink Cloud class, superstar learning, i-guidance, wisdom tree, UMU interactive learning platform, rain classroom, etc., each of the teaching platform related functions, use process and portability have their own advantages and disadvantages. Take Superstar Learning as an example, it has the advantages of massive resources and data synchronization, but there are also defects excessive software memory usage and fluency in use.

Each student used at least 4 teaching platforms per semester, and online course designers often bring resources accumulated over many years of teaching to the teaching platform. The teaching presentation method is too rich, for the learners to form a number of highly centralized information resource libraries. Faced with a large amount of redundant information, it is impossible to attract learners to build cognitive focus, deal with this irrelevant information, and also occupy the limited working memory of the learners, increasing the external cognitive load.

Hybrid design based on flipping classroom affects cognitive load. Because the professional basic knowledge, learning motivation and learning interest, cognitive ability and memory levels of different learning groups are significantly different, the traditional classroom teaching model has a single utility risk. In order to change this educational bottleneck, educators optimize the organizational form of learning materials, the way in which knowledge is presented, the interaction design of teaching content and the architectural schema effect between them. The hybrid teaching model based on flipping classroom emerges as the times require. Often combined with "MOOC + flip", "micro-course + flip", "MOOC + SPOC + flip”, the curriculum of different universities has become a characteristic online open course, which solves the aggregation of three-dimensional curriculum teaching resources. Sharing issues in order to achieve the goal of learning self-study, mutual learning and group learning. Instructional designers often split the series of thematic courses into a number of different teaching sessions. Although each teaching session reduces the learner' $\mathrm{s}$ internal cognitive load, the coherence is slightly insufficient. In the process of watching online courses, learners need to use limited working memory to retrieve past information knowledge and process and integrate various types of information, which will inevitably increase the external cognitive load.

\section{Control strategy of blended teaching models}

Build a schematized information space. Cognitive load theory believes that knowledge is stored in long-term memory in the form of schemas [5]. The main function of teaching is to store information in long-term memory. Schema organizes information based on how information elements are used. It provides a mechanism for knowledge organization and storage to reduce the workload of working memory. If the " $T$ " layout is adopted, the line of sight of the coherent learner can be effectively guided, and the overall cognitive load can be reduced. When it is necessary to emphasize the knowledge of individual information, it is suitable to adopt the disc structure or the histogram structure. However, the contrast, association, and interactive teaching tasks are more suitable for the use of line and scatter plot structures.

Enhance low cognitive load through information highlighting. Information highlighting refers to the process of visually capturing information, through information coding such as color, proportion, 
letters and numbers, element contrast to strengthen important information, weaken or remove interference information, that can reduce the degree of cognitive interference between visual [6]. For example, color hue, hue, lightness, purity, and saturation difference will affect the degree of highlighting. When using a dark background, warmer colors are more likely to produce information highlights than cold colors, by highlighting the important knowledge. By highlighting the annotation or marking of important knowledge, it helps to improve attention, enhance memory and improve cognitive performance. The shape feature, topological property, and complexity of the shape will affect the salience of the information. In the shape coding, we should avoid using multiple ring shapes as far as possible.

Filter secondary information to reduce cognitive load. The secondary information filtering aims to filter the knowledge points that are not related to the learner in a large amount of information flow according to the learners' knowledge acquisition requirements and use specific technical methods to reduce the number of learning materials and meet the learners' needs. Information is displayed to reduce the cognitive burden of learners and improve the efficiency of knowledge acquisition. Based on the principle of secondary information filtering, in the multimedia and visual teaching process, it should try to avoid too much knowledge information when each information is presented. It can weaken the secondary information, weaken the display background, reduce the text, and increase the graphics information. The content of knowledge presentation is more simple, reducing the thinking space of the learner and the space for the knowledge conversion work. For example, control color to represent different levels of information, control different shapes to express quantitative or qualitative knowledge content, the faster the learning speed and the less the number of colors or shapes should be controlled.

\section{Conclusions}

In the blended teaching activities, the frustration caused by the difficulty of the learners' knowledge absorption is too large, or the learning slackness caused by the too simple knowledge acquisition. In the final analysis, the imbalance of the brain cognitive load is too high or too low in the process of teaching interaction. In order to effectively improve the practical value of the blended teaching model, this paper analyzes the interaction process of "Knowledge-Cognition" from the perspective of learner cognitive load and brain information processing and points out that the main factor affecting classroom cognitive load comes from learning. The interaction between the environment, tasks, materials, and learners' own characteristics. Therefore, according to the limitations of cognitive resources and cognitive schema principles, the teaching design method is improved to balance the cognitive load of learners.

\section{Acknowledgements}

This work was financially supported by the East University of Heilongjiang Foundation (2018), Heilongjiang Education Science "13th Five-Year" Plan Project (GBC1317121) and Heilongjiang Education Teaching Reform Project (2018).

\section{References}

[1] Ying, F. U. Jornal of N of Ahr Ollg \& Nvry (2012).

[2] Sweller, J. Cognitive Science (1994).

[3] Sweller, John. Educational Psychology Review. Vol. 22.2(2010), p. 123-138.

[4] Shannon, C. E. Bell System Technical Journal. Vol. 27.4(1948), p. 379-423.

[5] Ericsson K A, Kintsch W. Psychological Review. Vol. 120.2(1995), p. 211-245.

[6] Vytal, Katherine, et al. Psychophysiology. Vol. 49.6(2012), p. 842-852. 